Improvement of health care delivery to minimize disruptions is another important area that requires more research. ${ }^{1}$ Power outages during extreme heat can create dangerous situations in which patients may lose access to cooling equipment or electricitydependent medical supplies at home. Hospitals must rely on backup generators that may power only certain aspects of operation, resulting in technological complications and turning normally high-tech hospitals into limitedresource environments. Recently, nearly 250 hospitals were affected by the intentional power outages in California, undertaken to reduce the risk of wildfires. Many of the events that expose us to the effects of climate change can also result in supply-chain disruptions like those that have caused shortages of intravenous saline. ${ }^{1}$ Such disruptions further hinder clinicians' ability to provide care, and they present a significant opportunity to proactively prepare instead of reflexively reacting to each individual crisis.

Despite the irony, I often describe our current knowledge of the health effects of climate crisis as an iceberg. Though we see a peak above the water's surface, there is much more to fear from the larger mass beneath - the effects that we haven't yet identified. For example, rising temperatures were recently linked to increasing bacterial resistance to antibiotics. ${ }^{5}$ The full health implications of the climate crisis may be far more immense and insidious than we have so far imagined. Although dedicated climate and health research is needed, this gap can be addressed more rapidly by adding a climate-change lens to existing lines of research.

Transitioning from theoretical discussions to practical applications will require multidisciplinary collaboration and sharing of best practices. We will need to learn from health professionals and systems that have already been facing dynamic climate threats that will increasingly affect other regions. Collaboration is the driving force behind the Climate Crisis and Clinical Practice initiative that is being launched in Boston on February 13, 2020, with the first of what we, the organizers, hope will be numerous symposia held throughout the United States and elsewhere. The initiative aims to highlight this critical need and provide an online forum to promote conversation. Although ulti- mately the best medicine for the climate crisis is preventive the urgent reduction of greenhouse gases - we cannot ignore the myriad ways in which our patients' health is already being harmed and our responsibility to improve our practice.

Disclosure forms provided by the author are available at NEJM.org.

From the Department of Emergency Medicine, Massachusetts General Hospital, Harvard Medical School, and the Center for Climate, Health, and the Global Environment, Harvard T.H. Chan School of Public Health, Boston, and the Harvard Global Health Institute, Cambridge - all in Massachusetts.

1. Salas RN, Solomon CG. The climate crisis - health and care delivery. N Engl J Med 2019;381(8):e13.

2. Vaidyanathan A, Saha S, Vicedo-Cabrera $\mathrm{AM}$, et al. Assessment of extreme heat and hospitalizations to inform early warning systems. Proc Natl Acad Sci U S A 2019;116: 5420-7.

3. Nogueira LM, Sahar L, Efstathiou JA, Jemal A, Yabroff KR. Association between declared hurricane disasters and survival of patients with lung cancer undergoing radiation treatment. JAMA 2019;322:269-71.

4. Hoye WL, Mogalian EM, Myrdal PB. Effects of extreme temperatures on drug delivery of albuterol sulfate hydrofluoroalkane inhalation aerosols. Am J Health Syst Pharm 2005;62:2271-7.

5. MacFadden DR, McGough SF, Fisman D, Santillana M, Brownstein JS. Antibiotic resistance increases with local temperature. Nat Clim Chang 2018;8:510-4.

DOI: 10.1056/NEJMp2000331

Copyright $\odot 2020$ Massachusetts Medical Society.

\title{
Modernizing Scope-of-Practice Regulations - Time to Prioritize Patients
}

\author{
Bianca K. Frogner, Ph.D., Erin P. Fraher, Ph.D., M.P.P., Joanne Spetz, Ph.D., Patricia Pittman, Ph.D., \\ Jean Moore, Dr.P.H., Angela J. Beck, Ph.D., M.P.H., David Armstrong, Ph.D., and Peter I. Buerhaus, Ph.D., R.N.
}

$\mathrm{O}$ ngoing payment reforms are pressing health systems to reorganize delivery of care to achieve greater value, improve access, integrate patient care among settings, advance population health, and address social determinants of health. Many organizations are experimenting with new ways of unleashing their workforce's potential by using telehealth and various forms of digital technology and developing team- and community-based delivery models. Such approaches require reconfiguring of provider roles, but states and health care organizations often place restrictions on health professionals' scope of practice that limit their flexibility. ${ }^{1}$

These restrictions are inefficient, increase costs, and reduce access to care. As leaders of public and private research centers 
who interact with and study the U.S. health workforce, we believe it's time to revise the country's antiquated patchwork of laws that restrict the health system's ability to innovate. We should improve our approach to regulating health professionals' scope of practice so that regulations better serve the needs of patients, rather than protect turf in the battles among health professions.

State licensure boards determine which health care practitioners are licensed, the requirements for obtaining licensure, and what services various practitioners can provide. As a result, the services performed by members of the same health profession may vary widely among states. An additional layer of regulation occurs at the health care organization level, where privileges are determined by medical-staffing committees and other leadership bodies. Policymakers are increasingly recognizing that differences in state laws and in the ways in which organizations deploy their workforces aren't based on evidence regarding quality of care or safety. Rather, state laws and organizational policies are informed by lobbying by professional associations that jockey to impose their self-interested views. ${ }^{2,3}$

There are two major consequences associated with restricting the scope of practice of qualified and competent workers who have been trained to safely and efficiently provide services: skills aren't used to their full extent, and workers aren't employed in innovative ways to meet health care needs. The status quo is unproductive, wasteful, and costly. Psychiatric pharmacists, for example, could help offset the shortage of psychiatrists by providing medication-management services. In addition, many states don't al- low these practitioners to prescribe buprenorphine, despite the need for more trained clinicians to mitigate the opioid epidemic. Dental therapists provide routine preventive and restorative oral health care services, including preparation and filling of cavities. Although dental therapists or equivalent practitioners augment the capacity of the oral health workforce in at least 50 countries and a vast body of evidence supports the safety and effectiveness of this approach, professional dentists' organizations continue to oppose legislation to authorize dental therapists to practice in the United States. Similarly, home care aides, who provide assistance with activities of daily living for millions of frail older adults and younger people living with disabilities, are subject to regulations that reduce their ability to meet clients' care needs. In many states, licensed nurses are prohibited from delegating various tasks to aides, including administration of routine medications. We are unaware of evidence that such restrictions protect patient safety. On the contrary, there is growing evidence that expanded delegation benefits patients.

Traditional workforce-planning approaches have imposed similar constraints by trying to identify the "right" number of each type of health professional needed in the future. Most health workforce models have taken a silo-based approach that assumes that each health profession has an exclusive and fixed scope of practice. Contemporary workforce-planning models have begun to transition away from these profession-centered approaches toward population-based approaches that start with different questions: What are the population's health care needs? And how might fully enabled teams of providers meet these needs? ${ }^{4}$ Such an approach requires shifting from a focus on provider shortages to a recognition that health professions have scopes of practice that overlap and can, if regulation allows, adapt depending on patients' health care needs and on other members of the care team. ${ }^{4}$ New workforce models for behavioral health needs, for example, could include social workers and community health workers in addition to psychiatrists, psychiatric nurse practitioners, and physician assistants.

Regulators can change the ways in which scope-of-practice regulations are created and revised by making decisions on the basis of evidence regarding quality and safety, rather than the objections raised by other health professions. Strategies for increasing the use of evidence in decisions about scope of practice include implementing state-based requirements for in-depth policy analysis, issuing "sunrise" reports that document the need for proposed changes, estimating the costs and benefits to the public of such changes, and assessing potential alternatives. When insufficient evidence is available to support a change, demonstration programs such as California's Health Workforce Pilot Project, which permits testing and rigorous evaluation of changes in scopes of practice, may be indicated. ${ }^{5}$

Although each state has the authority to establish scope-ofpractice regulations, we believe it's time to standardize evidencebased minimum scopes of practice for health professionals. Greater uniformity would support health professionals' ability to practice to the full extent of their education and training and enhance opportunities for efficient and effective health service delivery that 
better meets patients' needs. Uniformity is especially important for the provision of telehealth services, since implementation can be hindered by state scope-of-practice rules that restrict practitioners from working across state lines. Expanded use of interstate licensing compacts would also support more effective and more efficient telehealth service delivery.

Educators in the health professions also have an important role in modernizing scope-of-practice regulations. Traditional programs that educate health professionals in silos reinforce restrictive approaches. We believe it's important to shift to a focus on interprofessional collaboration in practice environments that support continuous learning about how best to serve patients. Interprofessional education can help learners understand the histories, perspectives, and contributions of various professions and better prepare health care professionals to work in teams.

Finally, clinicians can raise questions and challenge their professional associations, state regulatory bodies, insurance companies, and leaders in charge

An audio interview with Dr. Frogner is available at NEJM.org of making decisions about scope of practice in health care delivery organizations. Even in states that permit more expansive scopes of prac- tice, many health care delivery organizations are slow to allow expanded staff privileges in accordance with reforms. Clinical and administrative leaders within health care organizations can discuss the ways in which such restrictions affect efficiency, costs, and the configuration of teams and what changes could be made to better meet patients' needs.

Over the past decade, numerous reforms have been implemented by the federal government and by states to expand health insurance coverage, change payment models, motivate organizations to reconfigure the ways they deliver care, modify eligibility for Medicaid, and better prepare the health workforce for pressing behavioral care, primary care, geriatric care, and community care needs. To realize the potential of these laudable reforms, we believe that states should eliminate overly restrictive scope-of-practice regulations that they impose on the health professions. Doing so would allow us to unlock the full potential of the country's health workforce.

Disclosure forms provided by the authors are available at NEJM.org.

From the Center for Health Workforce Studies and the Department of Family Medicine, School of Medicine, University of Washington, Seattle (B.K.F.); the Carolina Health Workforce Research Center and the Department of Family Medicine, University of North Carolina at Chapel Hill, Chapel Hill (E.P.F.); the Health Workforce Research
Center on Long-Term Care and the Philip R. Lee Institute for Health Policy Studies, University of California, San Francisco, San Francisco (J.S.); the Fitzhugh Mullan Institute for Health Workforce Equity, Milken Institute School of Public Health, George Washington University, Washington, DC (P.P.); the New York Center for Health Workforce Studies (J.M.) and the Workforce Technical Assistance Center (D.A.), University at Albany-SUNY School of Public Health, Rensselaer; the Behavioral Health Workforce Research Center and School of Public Health, University of Michigan, Ann Arbor (A.J.B.); and the Center for Interdisciplinary Health Workforce Studies and the College of Nursing, Montana State University, Bozeman (P.I.B.).

1. Federal Trade Commission. Policy perspectives: competition and the regulation of advanced practice nurses. March 2014 (https://www.ftc.gov/system/files/documents/ reports/policy-perspectives-competition -regulation-advanced-practice-nurses/ 140307aprnpolicypaper.pdf).

2. McMichael B. The demand for healthcare regulation: the effect of political spending on occupational licensing laws. South Econ J 2017;84:297-316.

3. Department of Health and Human Services, Department of the Treasury, Department of Labor. Reforming America's healthcare system through choice and competition. December 2018 (https://www.hhs.gov/sites/ default/files/Reforming-Americas-Healthcare -System-Through-Choice-and-Competition .pdf).

4. Fraher E, Brandt B. Toward a system where workforce planning and interprofessional practice and education are designed around patients and populations not professions. J Interprof Care 2019;33:389-97.

5. Wides C, Dower C. A review of California Office of Statewide Health Planning and Development, Health Workforce Pilot Projects Program 1973-2007. San Francisco: Healthforce Center at UCSF, May 2010 (https:// healthforce.ucsf.edu/publications/review -california-office-statewide-health-planning -and-development-health-workforce).

DOI: $10.1056 /$ NEJMp1911077

Copyright (C) 2020 Massachusetts Medical Society.

\title{
On Suboptimization - Cadillac Care at the Mecca
}

\author{
Brendan M. Reilly, M.D.
}

fter David had a stent put in his bile duct, the Tumor Board said he needed a Whipple procedure, but 3 weeks later the surgeon hadn't scheduled him, and a friend whose uncle died of pancreatic cancer said David should go to the best place. When you get on an airplane, she said, you want $a$ pilot who does this every day. So they called three famous cancer centers and interviewed surgeons who do Whipples all the time. David hoped for the place where it doesn't snow, but that guy, when asked about his operative mortality, got huffy and hung up. The second place didn't "target the tumor" as Google recommended, so David favored the place whose surgeon agreed to see him immediately. 